\title{
MATRIX METHODS AND THE PROPERTY OF STRETCHINGS
}

\author{
T. A. KEAGY
}

(Communicated by J. Jerry Uhl, Jr.)

\begin{abstract}
D. F. Dawson has proved that if $x$ is a sequence and $A$ is a matrix with convergent row sums, then there exist a stretching $z$ of $x$ and a row finite matrix $B$ such that $A y$ and $B y$ converge or diverge together for each stretching $y$ of $z$. An extension of this result is used to answer a question proposed by D. Gaier regarding the conditions necessary for a matrix $A$ to have the property that if $x$ is a sequence with finite limit point $t$, then $A$ sums a stretching of $x$ to $t$.
\end{abstract}

1. Introduction. The matrix $A$ is a regular summability method if $A$ sums every convergent sequence $x$ to $\lim _{n} x_{n}$. The familiar Silverman-Toeplitz characterization of regular matrices consists of the following three conditions:

(a) $\lim _{p} a_{p q}=0$ for $q=1,2,3, \ldots$,

(b) $\lim _{p} \sum_{q} a_{p q}=1$, and

(c) $\sup _{p} \sum_{q}\left|a_{p q}\right|$ is finite.

The sequence $y$ is a stretching of $x$ if there exists an increasing sequence of positive integers $(\kappa(n))_{n=1}^{\infty}$ such that $y_{i}=x_{1}$ if $i \leq \kappa(1)$ and $y_{i}=x_{j}$ if $\kappa(j-1)<$ $i \leq \kappa(j)$ for $j>i$. For each stretching $y$ of $x$ there exists a regular matrix $S$ with all entries either 0 or 1 such that $S x=y$.

In [1] D. F. Dawson uses the following lemma to help characterize those matrices which sum every stretching of some fixed divergent sequence. In $\S 2$ we extend the lemma to answer a question proposed by D. Gaier [3] regarding the conditions necessary for a regular matrix $A$ to have the property that if $x$ is a sequence with finite limit point $t$, then there exists a stretching $y$ of $x$ such that $\lim A y=t$.

LEMMA 1 (DAWSON). If $x$ is a sequence and $A$ is a matrix with convergent row sums, then there exist a stretching $z$ of $x$ and a row finite matrix $B$ such that if $y$ is any stretching of $z$, then $A y$ and By converge or diverge together.

2. The property of stretchings. D. Gaier [3] has studied the necessary and sufficient conditions for a real-valued matrix $A$ to have the property that if $x$ is a sequence in $\mathbf{R}^{n}$ (with the usual Euclidean norm) and $t$ is a finite limit point of $x$, then $A$ maps some stretching of $x$ into a sequence that converges to $t$. (Similar questions regarding $(C, 1)$ summability and real-valued sequences have been considered by V. Drobot [2] and J. Isbell [4].)

Received by the editors March 15, 1986. Presented to the AMS at the 825 meeting in New Orleans, Louisiana.

1980 Mathematics Subject Classification (1985 Revision). Primary 40C05.

Key words and phrases. Regular, segment, stretching. 
Gaier calls the set $S=\left\{k_{1}, k_{1}+1, \ldots, k_{2}\right\}$ of consecutive positive integers a segment of the matrix $A$ with beginning $k_{1}$ and end $k_{2}$. He defines

$$
Z(S)=\sup _{p}\left|\sum_{q \in S} a_{p q}\right| .
$$

The notation $S=\bigcup_{j=1}^{n} S_{j}$ when referring to segments means the segment $S$ is formed by the union of $n$ nonempty adjacent subsegments.

The matrix is said to have property (G) if for each triple $(\varepsilon, k, K)(\varepsilon>0 ; k, K$ positive integers) there exists a segment $S$ with beginning greater than $K$ such that $S=\bigcup_{j=1}^{k} S_{j}$ and $Z\left(S_{j}\right)<\varepsilon$ for $j=1,2,3, \ldots, k$. It is easy to see there exist regular matrices (e.g. the identity matrix) which do not satisfy property $(G)$.

The matrix $A$ is said to have the property of stretchings if, for each sequence $x$ and each finite limit point $t$ of $x$, there exists a stretching $y$ of $x$ such that $\lim A y=t$. The following two results are due to Gaier [3].

THEOREM 1 (GAIER). If $A$ is a regular matrix satisfying $(\mathrm{G})$, then $A$ has the property of stretchings.

THEOREM 2 (GAIER). If $B$ is a row-finite regular matrix which does not satisfy property $(\mathrm{G})$, then for certain dimensions $n, B$ does not have the property of stretchings.

The proof of Theorem 2 provided by Gaier makes use of the fact $B$ is row finite. In his paper, Gaier questions whether row finiteness is in fact a necessary condition for the theorem to be true. In order to answer that question we provide an extension to Lemma 1 that differs from Dawson's original result in at least one significant way. Lemma 1 requires knowledge of both $A$ and $x$ before the row finite matrix can be identified. The following result only requires a knowledge of $A$ before identifying $B$.

THEOREM 3. Let $A$ be a regular matrix that does not satisfy property (G). There exists a row finite regular matrix $B$ that does not satisfy property $(\mathrm{G})$ such that every sequence $x$ has a stretching $z$ with the property that $A y-B y$ is null for each stretching $y$ of $z$.

PROOF. Assume $n_{1}<n_{2}<\cdots<n_{k-1}$ have been chosen and let $n_{k}>n_{k-1}$ such that $\left|\sum_{q=r}^{s} a_{p q}\right|<2^{-k} k^{-1}$ for every $n_{k} \leq r \leq s$ and $p \leq k$. Define $b_{k q}=a_{k q}$ if $q<n_{k}$ and $b_{k q}=0$ otherwise. Clearly $B$ satisfies properties (a) and (c) of regularity. Since for each $p$

$$
\begin{aligned}
\left|\sum_{q=1}^{\infty} a_{p q}-\sum_{q=1}^{\infty} b_{p q}\right| & =\left|\sum_{q=n_{p}}^{\infty} a_{p q}\right| \\
& \leq \sum_{i=p}^{\infty}\left|\sum_{q=n_{i}}^{n_{i+1}-1} a_{p q}\right| \\
& <2^{-p+1},
\end{aligned}
$$

the matrix $B$ also satisfies property (b) of regularity. 
Since $A$ does not satisfy property (G), there exist $(\varepsilon, k, K)$ such that every segment $\bigcup_{j=1}^{k} S_{j}$ of $A$ with beginning greater than $K$ has some $1 \leq i \leq k$ such that $Z\left(S_{i}\right) \geq \varepsilon$. Let $K^{\prime}=\min \left\{n_{p} \geq K: 1 / 2^{p}(p+1)<\varepsilon / 3\right\}$. Let $\bigcup_{j=1}^{k} S_{j}$ be any segment of $B$ with beginning greater than $K^{\prime}$. Let $1 \leq i \leq k$ such that $Z\left(S_{i}\right) \geq \varepsilon$ for the matrix $A$. Let $k_{1}$ and $k_{2}$ be the beginning and end of $S_{i}$ and let $p$ be a row of $A$ with $\left|\sum_{q=k_{1}}^{k_{2}} a_{p q}\right|>2 \varepsilon / 3$. By the definitions of $K^{\prime}$ and $n_{p}$, it follows that $k_{1} \leq n_{p}$. If $k_{1}<n_{p} \leq k_{2}$, then

$$
\left|\sum_{q=k_{1}}^{k_{2}} b_{p q}\right| \geq\left|\sum_{q=k_{1}}^{k_{2}} a_{p q}\right|-\left|\sum_{q=n_{p}}^{k_{2}} a_{p q}\right|>\frac{2 \varepsilon}{3}-\frac{\varepsilon}{3}=\frac{\varepsilon}{3} .
$$

If $k_{2}<n_{p}$, then

$$
\left|\sum_{q=k_{1}}^{k_{2}} b_{p q}\right|=\left|\sum_{q=k_{1}}^{k_{2}} a_{p q}\right|>\frac{2 \varepsilon}{3}
$$

Because $B$ has no segment $\bigcup_{j=1}^{k} S_{j}$ with beginning greater than $K^{\prime}$ and $Z\left(S_{i}\right)<\varepsilon / 3$ for $1 \leq i \leq k, B$ does not satisfy property (G).

Let $x$ be a sequence and define

$$
\begin{aligned}
& \kappa(1)=\min \left\{n_{p}: \sum_{j=1}^{2}\left\|x_{j}\right\|<p\right. \text { and } \\
& \left.\qquad\left\|x_{2}\right\|\left|\sum_{q=r}^{s} a_{u q}\right|<2^{-2} \text { for } u \leq p \text { and } n_{p} \leq r \leq s\right\} .
\end{aligned}
$$

In general let

$$
\begin{aligned}
& \kappa(k)=\min \left\{n_{p}<\kappa(k-1):\right. \sum_{j=1}^{k+1}\left\|x_{j}\right\|<p \text { and } \\
&\left.\left\|x_{k+1}\right\|\left|\sum_{q=r}^{s} a_{u q}\right|<2^{-(k+1)} \text { for } u \leq p \text { and } n_{p} \leq r \leq s\right\} .
\end{aligned}
$$

Let $z$ be the stretching of $x$ determined by $\kappa$. Let $y$ be a stretching of $z$. Then $y$ is a stretching of $x$ determined by some sequence $\sigma$ satisfying $\kappa(k) \leq \sigma(k)$ for each $k$.

Consider first a row $p$ with $n_{p} \leq \kappa(1)$. Then

$$
\begin{aligned}
\left\|\sum_{q=n_{p}}^{\infty} a_{p q} y_{q}\right\| & \leq\left\|x_{1}\right\|\left|\sum_{q=n_{p}}^{\sigma(1)} a_{p q}\right|+\sum_{i=2}^{\infty}\left\|x_{i}\right\|\left|\sum_{q=\sigma(i-1)+1}^{\sigma(i)} a_{p q}\right| \\
& <\left\|x_{1}\right\|\left|\sum_{q=n_{p}}^{\sigma(1)} a_{p q}\right|+\sum_{i=2}^{\infty} 2^{-i}
\end{aligned}
$$


and $(A y)_{p}$ converges. Next assume there exists some $j \geq 1$ such that $\kappa(j)<n_{p} \leq$ $\kappa(j+1)$, then $\sum_{i=1}^{j+1}\left\|x_{i}\right\|<p$ and

$$
\begin{aligned}
\left\|(A y)_{p}-(B y)_{p}\right\| & =\left\|\sum_{q=n_{p}}^{\infty} a_{p q} y_{q}\right\| \\
& \leq\left[\sum_{i=1}^{j+1}\left\|x_{i}\right\|\right] 2^{-p} p^{-1}+\sum_{i=j+2}^{\infty}\left\|x_{i}\right\|\left|\sum_{q=\sigma(i-1)+1}^{\sigma(i)} a_{p q}\right| . \\
& <2^{-p}+2^{-(j+1)}
\end{aligned}
$$

Because $j$ increases with $p, A y-B y$ is null and the proof is complete.

Although the statement provided in Theorem 3 will be sufficient to answer the question proposed by Gaier regarding the necessity of row finiteness in Theorem 2, the proof provided here actually establishes the following stronger result.

COROLLARY 1. Let $A$ be a matrix with convergent row sums that does not satisfy property $(\mathrm{G})$. There exists a row-finite matrix $B$ that does not satisfy property $(\mathrm{G})$ such that if $\left(H_{n}\right)$ is any nondecreasing positive term sequence, then there exists a stretching matrix $S$ such that $A y-B y$ is null whenever $x$ is a sequence with $s_{n}=\sum_{i=1}^{n}\left\|x_{i}\right\|$ and $s_{n}=O\left(H_{n}\right)$, and $y$ is a stretching of $S x$.

The following theorem settles the question of whether row finiteness is required in Theorem 2.

THEOREM 4. If $A$ is a regular matrix that does not satisfy property $(\mathrm{G})$, then for certain dimensions $n, A$ does not have the proeprty of stretchings.

Proof. Let $B$ be the row finite matrix described in Theorem 3. By Theorem 2 , for certain dimensions $n, B$ does not satisfy the property of stretchings. Let $x$ be a sequence from $\mathbf{R}^{n}$ with finite limit point $t$ such that $B$ sums no stretching of $x$ to $t$. By Theorem 3 there exists a stretching $z$ of $x$ such that $A y-B y$ is null for each stretching $y$ of $z$. Since $B$ sums no stretching of $x$ to $t$, it follows that $B$ sums no stretching of $z$ to $t$. Hence $A$ sums no stretching of $z$ to $t$, and $A$ does not have the property of stretchings for dimension $n$.

\section{BIBLIOGRAPHY}

1. D. F. Dawson, A Tauberian theorem for stretchings, J. London Math. Soc. (2) 13 (1976), 27-33.

2. V. Drobot, On the dilution of series, Ann. Polon. Math. 30 (1975), 323-331.

3. D. Gaier, Limitierung gestreckter Folgen, Publ. Ramanujan Inst. No. 1, (1969), 223-234.

4. J. Isbell, On dilution and Cesàro summation, Proc. Amer. Math. Soc. 45 (1974), 397-400.

Department of Mathematics, The University of texas at Tyler, Tyler, TEXAS 75701

Current address: Department of Mathematics and Computer Science, Duquesne University, Pittsburgh, Pennsylvania 15282 